\section{PROTEINS}

\section{Ends and Enzymes}

from our Molecular Biology Correspondent

THE evolution of solid state techniques has transformed the craft of peptide synthesis into a relatively routine, or at least not too intimidating, operation. This development has opened the way to a variety of diverting stratagems, such as elimination or replacement of individual side chains in enzymes to see what happens to the structure and activity. The state of the art is not yet, of course, such as to encourage any lighthearted attempts at synthesizing whole enzymes. What is needed, rather, is an enzyme from which an interesting oligopeptide can be reversibly detached after specific cleavage of the chain at a single point. One can then shuffle the permutations of composition, sequence and length of synthetic analogues of the oligopeptide. This strategy has been pursued to great effect by Anfinsen and his colleagues on staphylococcal nuclease, which can be assembled non-covalently from various fragments, and by Scoffone and Hoffmann and their co-workers on the pancreatic ribonuclease $S$-protein-S-peptide system. The S-peptide comes from the $\mathrm{N}$-terminus, and is prepared by exposure of the enzyme to subtilisin. It has recently been found that under the action of pepsin a $\mathrm{C}$-terminal peptide can be split off the other end of the chain, again with reversible loss of enzymatic activity.

Lin et al. (J. Biol. Chem., 247, 4763 ; 1972) have now explored the effects of changes in size and composition of the terminal fragment, and of the length of the residual ribonuclease chain, on binding and reactivation of the catalytic activity. Pepsin breaks the chain between residues 120 and 121 , so that the last four residues of the native enzyme are eliminated. Carboxypeptidase can then be used to nibble away one or two further residues from the end, the second of which is the active site constituent, his-119. The truncated enzyme is inactive, and can be reactivated by addition of overlapping peptides of different lengths. The first curious observation is that C-terminal peptides containing fewer than nine amino-acids are capable of inducing only traces of catalytic activity. When the nonapeptide (116-124) is added to the core $(1-118)$, the activity jumps to 60 per cent of native, and as the length of the peptide is increased so the activity rises still further, reaching 98 per cent for the complex of 1.118 with 111-124. The same pattern (though with slightly lower activities) emerged when the residual chain was one or two residues longer. The activities, it should be noted. do not depend on the strength of the protein-peptide association, for they refer to saturation conditions with a large molar excess of peptide. The association constant can be independently evaluated by measuring the activity as a function of mole ratio of peptide to protein, and was found also to rise with increasing length of the peptide. Evidently then the overlapping residues in the peptide interact in an energetically desirable manner with the protein.

Lin et al. have, moreover, hit on a nice method of establishing whether it is the side chains of the protein or the peptide that participate in the globular conformation, in particular which of them contributes the active centre his-119 side chain. The criterion is that, in the native state, his-119 has greatly enhanced reactivity towards alkylating agents. Ribonuclease 1-120, for example, which has very little enzymatic activity, also has low reactivity at this residue. The complexes were accordingly carboxymethylated with iodoacetic acid, and the extent of carboxymethylation in the protein and peptide determined. With complexes involving ribonuclease 1-120, the protein histidine reacts preferentially by a factor of four, whereas with 1.119 the ratio is $1: 1$. Thus, as in a similar complex of staphylococcal nuclease fragments, there are two active configurations of comparable energy.

In a further study, Lin et al. (ibid., 4768) have examined the importance of the residue phe-120, which lies close to the substrate pyrimidine ring, and, as judged by n.m.r. experiments, seems to interact with it. Peptides with fourteen residues were synthesized, in which the phenylalanine was variously replaced. With leucine or isoleucine in its place, the enzymatic activity and the association constant of peptide and protein were both reduced, but only by one order of magnitude. This level of activity is correlated with the reactivity of his-119. When the phenylalanine was replaced by tryptophan, activity was down by two orders of magnitude. Evidently then there is no enzymatically important interaction of the phenyl ring with the pyrimidine of the substrate. Instead it must be presumed to be implicated in a hydrophobic cluster, which stabilizes this part of the chain, and probably keeps his-119 properly oriented in the active centre. The indole is evidently too big to fit into the slot without distortion. A particularly striking feature, which lends support to this general picture, is that in spite of the lower activity of the leucine and isoleucine analogues, the Michaelis constants for hydrolysis of cyclic CMP, and also the binding constant for the inhibitor 2'-CMP, are indistinguishable from values for the enzyme containing phenylalanine. The authors suggest that the impaired activity of the analogues may result from imprecisions in the geometry of the active site.

A similar approach has been adopted by Katsoyannis and Zalut (Biochemistry, 11, 3065; 1972) to determine

\title{
Hybrid Cells and DNA Repair
}

WHAT should prove to be a very useful new approach to the problem of elucidating the biochemistry and genetics of DNA repair in mammalian cells is described in next Wednesday's Nature New Biology by Goldstein and Lin. They have exploited the fact that fibroblasts from patients with the autosomal recessive genetic disease xeroderma pigmentosum are unable to repair their DNA after it has been damaged by ultraviolet irradiation, and are therefore easily killed by such irradiation to select hybrid cells one parent of which is a xeroderma pigmentosum fibroblast and the other a golden hamster cell lacking the enzyme hypoxanthineguanine phosphoribosyltransferase.

The karyotypes of these hybrid cells are very variable and change as the cells are repeatedly subcultured. After about forty-five generations twelve cells from one particular clone were examined in detail; the chromosome numbers of these cells ranged from 110 to 160 of which eight to sixteen chromosomes were identified as being from the human parental cell. Analysis of the glucose-6-phosphate dehydrogenase iso- zymes in cells of this and other clones of hybrids revealed both the human and the hamster molecules as well as a hybrid molecule.

Goldstein and Lin, having satisfied themselves that the cells which survived their selection procedure were indeed hybrids, then measured the ability of the hybrid cells to survive ultraviolet irradiation and to repair damaged DNA. About 50 per cent of hybrid cells and hamster parental cells survived doses of ultraviolet irradiation that allowed less than $10^{-4}$ of xeroderma pigmentosum cells to survive. Furthermore direct comparison of DNA repair activity after irradiation indicates that the hybrid cells have a repair activity intermediate between that of the two sorts of parental cells.

These data indicate that hamster repair enzymes can repair human DNA damaged by ultraviolet irradiation, and that they can partially compensate for the specific defect in xeroderma pigmentosum cells, namely excision repair. Obviously further exploitation of appropriate hybrid cells may help to piece together the biochemistry of this process. 